\title{
In-gap bound states and tunneling conductance of multiband superconductors through a normal/superconductor/superconductor junction
}

\author{
Xiao-Yong Feng and Tai-Kai Ng \\ Department of Physics, Hong Kong University of Science and Technology, Clear Water Bay Road, Hong Kong
}

(Received 9 December 2008; revised manuscript received 29 January 2009; published 4 May 2009)

\begin{abstract}
The tunneling conductance between a metal and a multiband $s$-wave superconductor with a thin layer of single-band $s$-wave superconductor sandwiched in between is examined in this paper. We show that an in-gap peak in conductance curve is found as a result of the formation of in-gap bound state between the single-band and multiband superconductor junctions if the phases of the superconducting order parameters of the multiband superconductor are frustrated. The implication of this result in determining the gap symmetry of the iron-based superconductors is discussed.
\end{abstract}

DOI: 10.1103/PhysRevB.79.184503

PACS number(s): 74.45.+c, 74.20.Rp

With the discovery of the iron-based (pnictides) superconductors, the superconductivity characterized by more than one order parameter, i.e., the multigap superconductors, becomes a hot topic. Band-structure calculations indicate that the material has a quasi-two-dimensional electronic structure, with four bands centered around the $\Gamma$ and $M$ points in the Brillouin zone, contributing to the Fermi surface. An immediate issue of interests is thus the symmetry of the superconductor order parameters. Besides the obvious possibility that all the bands share the same superconductor orderparameter symmetry, it is also possible that the order parameters pick up more complicated configurations because of the nontrivial Fermi-surface structure. For example, it has been proposed that the order parameters may have $s$-wave symmetry but with opposite sign between bands centered at $\Gamma$ and $M$ points. ${ }^{1-3}$ Other more exotic possibilities have also been proposed. ${ }^{4}$

Experimentally, the absence of $\pi$ phase shift between tunneling in different direction in scanning superconducting quantum interference device (SQUID) microscopy ${ }^{5}$ and the suppression of knight shift with decreasing temperature in NMR (Ref. 6) experiments seem to exclude spin-triplet pairing states. However controversy remains between the $d$-wave and the exotic $s$-wave symmetries. The $d$-wave symmetry gets support from NMR (Ref. 6) and lower critical-field data, ${ }^{7}$ indicating existence of nodes in the superconducting order parameter while the observations in angle-resolved photoelectron spectroscopy ${ }^{8,9}$ favor nodeless gaps.

Thus an experiment which can distinguish the different $d$-wave and $s$-wave gap symmetry possibilities is significant. In this paper, we show that the quasiparticle tunneling through a $N-S_{1}-S_{2}$ junction, where $N, S_{1}$, and $S_{2}$ represent normal metal, a single-band $s$-wave superconductor, and the targeted superconductor, respectively, provides a strong test to the above problem when tunneling data from different surfaces of target superconductor $S_{2}$ and with different thickness of $S_{1}$ are collected. The geometry of the junction is sketched in Fig. 1.

To begin with, we first review the situation of simple $s$-wave and $d$-wave superconductors. We shall assume that the superconductor order-parameter structure is prominent on the $x-y$ plane, and superconductivity along the $z$ direction mainly comes from Josephson effect, consistent with a quasi- two-dimensional band structure. For the s-wave superconductor, it is expected that the tunneling spectrum will be qualitatively similar, independent of the tunneling plane of the targeted superconductor and the thickness $(d)$ of $S_{1}$. The situation is, however, very different for the $d$-wave superconductor. The existence of a midgap state at the boundary between the $d$-wave superconductor and normal metal if the surface is cut along the (110) direction ${ }^{10}$ leads to a zero-bias peak in the tunneling spectrum along the (110) direction. Thus the existence of a zero-bias peak in tunneling experiment with one prominent surface orientation distinguishes between conventional $s$ - and $d$-wave superconductors. ${ }^{11}$

Next we consider a multiband $s$-wave superconductor with order parameters of opposite sign. We shall call it $\pm s$-wave superconductor in the following. It was pointed out in Ref. 12 that in-gap bound states generally exist at the interface between a single-band $s$-wave superconductor and a $\pm s$-wave superconductor, and it is expected that they will contribute to quasiparticle tunneling, leading to in-gap peaks in the tunneling conductance through an $N-S_{1}-S_{2}$ junction. We shall show in the following that the $\pm s$-wave superconductor has a rather unique tunneling spectrum that distinguishes itself from ordinary $s$ or $d$ wave superconductors.

We shall employ the semiclassical (WKB) approximation in our calculation where band-structure effects and interband scattering are largely neglected. This is justified because similar to midgap states in the (110) surface of a $d$-wave superconductor, the presence of in-gap states in the $S_{1}-S_{2}$ junction is protected by the topological structure of the problem and is not expected to be destroyed by these effects as long as they are not too strong. ${ }^{12}$ The validity of this approximation will be discussed in more detail at the end of this paper. The theory captures only the qualitative behaviors of the tunneling spectrum which are insensitive to the details of the microscopic Hamiltonian.

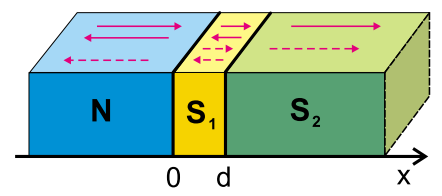

FIG. 1. (Color online) The geometry of the $N-S_{1}-S_{2}$ junction. The solid (dashed) arrows denote the propagation direction of particles (holes). 
We start with the Bogoliubov-de Gennes (BdG) equations ${ }^{13}$ for the quasiparticle spectrum,

$$
\epsilon^{(i)}\left(\begin{array}{c}
u^{(i)}(\vec{r}) \\
v^{(i)}(\vec{r})
\end{array}\right)=\left(\begin{array}{cc}
\hat{H}^{(i)}-\mu & \Delta^{(i)}(\vec{r}) \\
\Delta^{(i) *}(\vec{r}) & -\hat{H}^{(i)}+\mu
\end{array}\right)\left(\begin{array}{c}
u^{(i)}(\vec{r}) \\
v^{(i)}(\vec{r})
\end{array}\right),
$$

where $i=1, \ldots, N, N$ is the number of contributing bands in the multiband superconductor, $\hat{H}^{(i)}$ is the band dispersion of band $i$, and $\Delta^{(i)}(\vec{r})=\left|\Delta^{(i)}(x)\right| e^{i \theta^{(i)}(x)}$ is the corresponding superconductor order parameter. $\mu$ is the chemical potential. We have assumed that quasiparticles at different bands are decoupled from each other in writing down Eq. (1). The different bands are coupled only implicitly through the Josephson effect where ${ }^{13}$

$$
\Delta^{i}(\vec{r}) \sim-\sum_{j} V_{i j}\left\langle c_{\uparrow}^{(j)}(\vec{r}) c_{\downarrow}^{(j)}(\vec{r})\right\rangle,
$$

$\left\langle c_{\uparrow}^{(j)}(\vec{r}) c_{\downarrow}^{(j)}(\vec{r})\right\rangle$ is the pairing amplitude between electrons in $j$ th band, $V_{i i} \sim$ is the pairing interaction between electrons in band $i$, and $V_{i j}(j \neq i)$ is the Josephson coupling between order parameters in different bands. The relative phases between the order parameters in different bands is determined by the signs of $V_{i j}$ 's. ${ }^{12}$

To model the $N-S_{1}-S_{2}$ junction, we assume $\left[\left|\Delta^{(i)}(x, y, z)\right|, \theta^{(i)}(x, y, z)\right]=(0,0),\left(\Delta_{1}, 0\right)$, and $\left(\Delta_{2}^{(i)}, \theta^{(i)}\right)$ in the regions $x<0,0<x<d$, and $x>d$, respectively. The different regions are connected by $\delta$-function scattering potentials $U_{1} \delta(x)$ and $U_{2} \delta(x-d)$, respectively, and the system is assumed to be translational invariant in the $(y, z)$ direction. We also assume that the $\delta$-function scattering potentials do not introduce scattering between different bands, so that Eq. (1) remains valid in the presence of the junction. Within this approximation the total tunneling current is just the sum of tunneling currents from all bands. Notice that we are allowed to choose the phase of the single-band superconductor $S_{1}$ to be $\theta(0<x<d)=0$ since the overall phase of the system is a pure gauge. On the other hand there are in general $N$ different phases $\theta^{(i)}(x>d)$ in the multiband superconductor $S_{2}$. We shall assume that only two groups of $\theta^{(i)}$ 's exist, $\theta^{(i)}$ $=\left(\theta^{A}, \theta^{B}\right)$, with $\theta^{A}=\theta^{B}+\pi$ in the bulk multiband superconductor ( $\pm s$ wave), as proposed to be the case in the pnictide superconductors.

The Josephson coupling between a single-band superconductor and a $\pm s$-wave superconductor has been analyzed in Ref. 12. Let $T_{A(B)}$ be the Josephson coupling of the first (second) group of bands to $S_{1}$. It was found that when $T_{A}$ $\gg(\ll) T_{B}$, then $\theta_{A(B)}=0$ and $\theta_{B(A)}=\pi$. This state respects time-reversal symmetry and is called the TRI state. An alternative solution exists when $T_{A} \sim T_{B}$, at which case $\theta_{A}, \theta_{B}$ $\neq 0, \pi$. The state breaks time-reversal symmetry and is called the TRB state. The condition $T_{A} \sim T_{B}$ for the TRB state is satisfied only when there is a symmetry reason that $T_{A}$ and $T_{B}$ are (almost) equal to each other. For the Fe pnictides, this condition is satisfied for the electron pockets around the two $M$ points when the interface is perpendicular to the $\Gamma-X$ direction. When the interface is perpendicular to the $\Gamma-M$ direction, the hole pockets around the $\Gamma$ point and electron pockets around $M$ points are coupled to the $s$-wave superconductor with different tunneling strength, and hence TRB state is not expected. However, the in-gap states in the TRI state can offer the evidence for the opposite sign of the order parameters for electron and hole pockets as we shall see in this paper.

We first consider the tunneling conductance between the $s$-wave superconductor and band $i$ in the $\pm s$-wave superconductor with $\theta^{(i)}=\theta$. Equation (1) has plane-wave solutions of form

$$
\Psi(\vec{r})=\left(\begin{array}{c}
u(\vec{r}) \\
v(\vec{r})
\end{array}\right)=e^{i \vec{q}_{\|} \cdot \vec{r}} e^{i q x}\left(\begin{array}{c}
u_{q} \\
v_{q}
\end{array}\right),
$$

where we have neglected the band index $i$ for brevity. $u_{q}$ and $v_{q}$ satisfy

$$
\epsilon\left(\begin{array}{l}
u_{q} \\
v_{q}
\end{array}\right)=\left(\begin{array}{cc}
\xi_{\vec{q}} & \Delta \\
\Delta & -\xi_{\vec{q}}
\end{array}\right)\left(\begin{array}{l}
u_{q} \\
v_{q}
\end{array}\right),
$$

where $\xi_{\vec{q}}=\varepsilon_{\vec{q}}-\mu$ and $\varepsilon_{\vec{q}}$ is the band dispersion. We shall assume $\varepsilon_{\vec{q}}=\hbar^{2}\left(q^{2}+q_{\|}^{2}\right) / 2 m$ for all bands and $\mu=\hbar^{2} k_{f}^{2} / 2 m$ in our calculation where $k_{f}$ is the Fermi momentum and we shall consider the semiclassical limit $k_{f}, m \rightarrow \infty$ with $v_{f}$ $=\hbar k_{f} / m$, remaining finite such that band-structure effects are eliminated in our calculation, consistent with the assumption that there is no interband scattering in our calculation.

For a given energy $\epsilon$ and transverse momentum $q_{\|}$, there are four possible longitudinal momenta $q= \pm q_{+}, \pm q_{-}$, where $\hbar q_{+(-)}=\sqrt{2 m\left[\mu_{x}\left(q_{\|}\right)+(-) \sqrt{\epsilon^{2}-|\Delta|^{2}}\right]}$ and $\mu_{x}\left(q_{\|}\right)=\mu-\hbar^{2} q_{\|}^{2} / 2 m$. The corresponding $u_{q}$ and $v_{q}$ are given by $u_{q}\left(v_{q}\right)$ $=e^{+(-) i \theta / 2} \sqrt{\frac{1}{2}\left[1+(-) \frac{\xi_{q}}{\epsilon}\right]}$.

Applying these results to our junction problem the general scattering wave function of an incoming wave from metal side has the following form at various regions in space:

$$
\begin{array}{r}
\alpha \Psi_{N}(x)=\left(e^{i k_{+} x}+a_{1} e^{-i k_{+} x}\right)\left(\begin{array}{l}
1 \\
0
\end{array}\right)+a_{2} e^{i k_{-} x}\left(\begin{array}{l}
0 \\
1
\end{array}\right), \\
\alpha \Psi_{S_{1}}(x)=\left(a_{3} e^{i p_{+} x}+a_{4} e^{-i p_{+} x}\right)\left(\begin{array}{l}
u_{p_{+}} \\
v_{p_{+}}
\end{array}\right) \\
+\left(a_{5} e^{-i p_{-} x}+a_{6} e^{i p_{-} x}\right)\left(\begin{array}{l}
u_{p_{-}} \\
v_{p_{-}}
\end{array}\right), \\
\alpha \Psi_{S_{2}}(x)=a_{7} e^{i q_{+} x}\left(\begin{array}{c}
u_{q_{+}} \\
v_{q_{+}}
\end{array}\right)+a_{8} e^{-i q_{-} x}\left(\begin{array}{c}
u_{q_{-}} \\
v_{q_{-}}
\end{array}\right),
\end{array}
$$

where $\alpha=e^{-i \vec{q}_{\|} \cdot \vec{r}_{\|}}$and

$$
\begin{gathered}
\hbar k_{+(-)}=\sqrt{2 m\left[\mu_{x}\left(q_{\|}\right)+(-)|\epsilon|\right]}, \\
\hbar p_{+(-)}=\sqrt{2 m\left[\mu_{x}\left(q_{\|}\right)+(-) \sqrt{\epsilon^{2}-\left|\Delta_{1}\right|^{2}}\right]}, \\
\hbar q_{+(-)}=\sqrt{2 m\left[\mu_{x}\left(q_{\|}\right)+(-) \sqrt{\epsilon^{2}-\left|\Delta_{2}\right|^{2}}\right]} .
\end{gathered}
$$

Notice that $q_{\|}$is the same at all regions because of momentum conservation along the $y-z$ directions. However $\Delta_{2}$ $\rightarrow \Delta_{2}^{(i)}$ and $q_{+(-)}^{(i)}$ 's are in general different for different bands. 


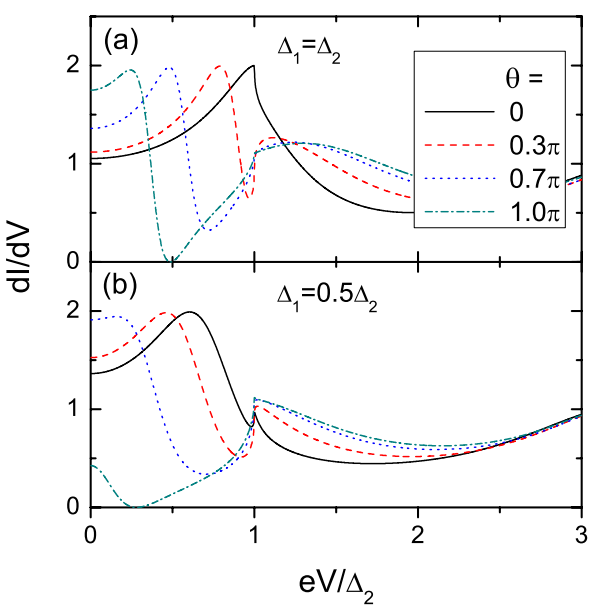

FIG. 2. (Color online) Tunneling conductance for various $\theta$ : (a) for $\Delta_{1}=\Delta_{2}=0.001 \mu$ and (b) for $\Delta_{1}=0.5 \Delta_{2}=0.0005 \mu$. $U_{1}=U_{2}=\mu$ and $d=\xi_{c 2}$ in both cases,.

The wave function has to satisfy the boundary conditions at the interfaces

$$
\begin{gathered}
\Psi_{N}(0)-\Psi_{S_{1}}(0)=0, \\
\Psi_{N}^{\prime}(0)-\Psi_{S_{1}}^{\prime}(0)=-\frac{2 m U_{1}}{\hbar^{2}} \Psi_{N}(0), \\
\Psi_{S_{1}}(d)-\Psi_{S_{2}}(d)=0, \\
\Psi_{S_{1}}^{\prime}(d)-\Psi_{S_{2}}^{\prime}(d)=-\frac{2 m U_{2}}{\hbar^{2}} \Psi_{S_{1}}(d),
\end{gathered}
$$

where the prime refers to the derivative with respect to the spacial coordinate $x$. The above equations determine the eight coefficients $\left\{a_{n}\right\}$ entering Eq. (4). The tunneling conductance defined by $d I / d V$ is proportional to $\int d q_{\|}\left\{k_{+}[1\right.$ $\left.\left.-\left|a_{1}(\epsilon=e V)\right|^{2}\right]+k_{-}\left|a_{2}(\epsilon=e V)\right|^{2}\right\}$. In the semiclassical limit $k_{f}$ $\gg q_{\|}$, the main contribution to the tunneling current comes from electrons with normal incidence to the interface. In this case we may approximate $k_{+} \sim k_{-} \sim k_{f}, \mu_{x} \sim \mu$, and the Blonder-Tinkham-Klapwijk (BTK) result

$$
\frac{d I}{d V} \sim 1-\left|a_{1}(\epsilon=e V)\right|^{2}+\left|a_{2}(\epsilon=e V)\right|^{2}
$$

is recovered. ${ }^{14}$ We shall employ this approximation in the following analysis.

To see the existence of bound states in the $S_{1}-S_{2}$ interface we first look at the $\theta$ dependence of the tunneling conductance. In Fig. 2 we plot $d I / d V$ versus $V$ for various values of $\theta$. We have chosen $\Delta_{2}=0.001 \mu, U_{1}=U_{2}=\mu$, and $d=\xi_{c 2}$, where $\xi_{c 2} \sim v_{f} / \Delta_{2}$ is the coherence length corresponding to the superconducting gap $\Delta_{2}$ in generating the figure. First we consider $\theta=0$ which represents tunneling between the usual (unfrustrated) $s$-wave superconductor junctions. In this case the conductance is peaked at the gap edges $\sim \Delta_{1}$ and $\Delta_{2}$, and there is no in-gap peak. The peak within the lower gap edge

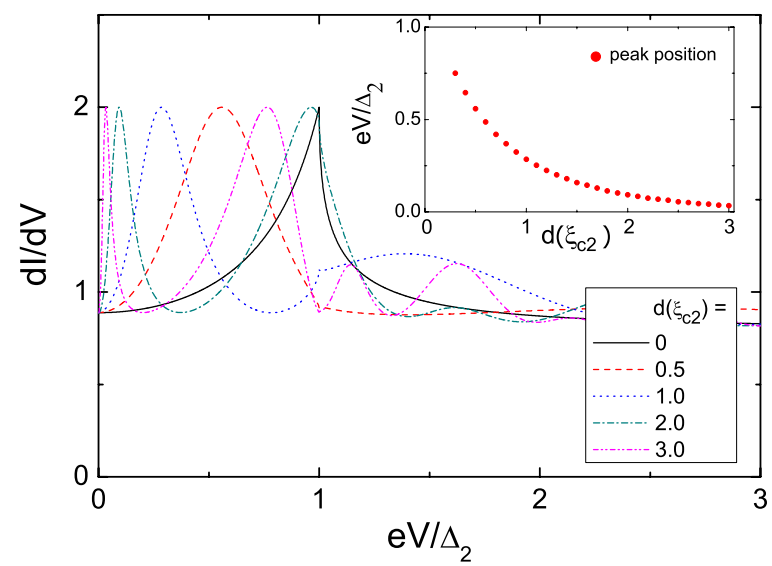

FIG. 3. (Color online) Tunneling conductance for various $d$. $\Delta_{1}=\Delta_{2}=0.001 \mu, U_{1}=\mu$, and $U_{2}=0$. The inset is the $d$ dependence of the conductance peak position.

moves toward zero energy as $\theta$ increases, corresponding to the appearance of TRB states. Notice that there is no conductance peak between $\Delta_{1}$ and $\Delta_{2}$.

Notice that instead of approaching the zero energy, the conductance peak saturates at a finite value $e V>0$ when $\theta$ $\rightarrow \pi$. This is because we have employed a finite thickness $d \sim \xi_{c 2}$ in our calculation. In this case the incoming and reflected waves inside $S_{1}$ interfere and split the zero-energy bound states into two states with the finite energy.

The thickness dependence of conductance is examined in Fig. 3 where we compute the tunneling spectrum for $\Delta_{1}$ $=\Delta_{2}$ and $\theta=\pi$ from $d=0$ to $d \sim 3 \xi_{c 2}$. When $d=0$, the tunneling behavior is the same as the case of normal-metal tunneling directly to a $s$-wave superconductor with no in-gap bound states. As $d$ increases, we see that an in-gap peak in conductance is developed with the peak position moving toward zero bias. The peak also becomes sharper as $d$ increases. The position of the peak as a function of $d$ is shown in the inset of Fig. 3 where it is clear that the peak position approaches zero exponentially as $d \rightarrow \infty .{ }^{12}$ Oscillatory behavior is also found in the conductance curves at energy $\mathrm{eV}$ $>\Delta_{1}$ at large $d$. This is a result of interference between multiple reflecting waves and is observable only if the total path length (approximately a few $d$ ) is smaller than the inelasticscattering mean-free path $(l)$ of the quasiparticles. We note that the existence of the in-gap conductance peak is independent of the surface orientation for an $\pm s$-wave superconductor. This and the $d$ dependence of the in-gap conductance peak provide a clear distinction between an ordinary $d$-wave and $\pm s$-wave superconductors.

The quantitative behavior of the tunneling conductance depends strongly on the scattering parameters $U_{1}$ and $U_{2}$ which depends on crystal surface orientation and other microscopic details of the system. Figure 4 shows the $d I / d V-V$ curves with various contact barrier $U_{1}$ and $U_{2}$ for $\theta=\pi$ and $\Delta_{1}=0.5 \Delta_{2}$. First we note that with $U_{1}$ fixed, the conductance is generally suppressed by $U_{2}$ at energy $e V>\Delta_{1}$. At this regime the quasiparticles do not see superconductor $S_{1}$ and the tunneling spectrum is qualitatively the same as the one from a normal metal directly to a $s$-wave superconductor. We also observe that the conductance peak within $\Delta_{1}$ originating 


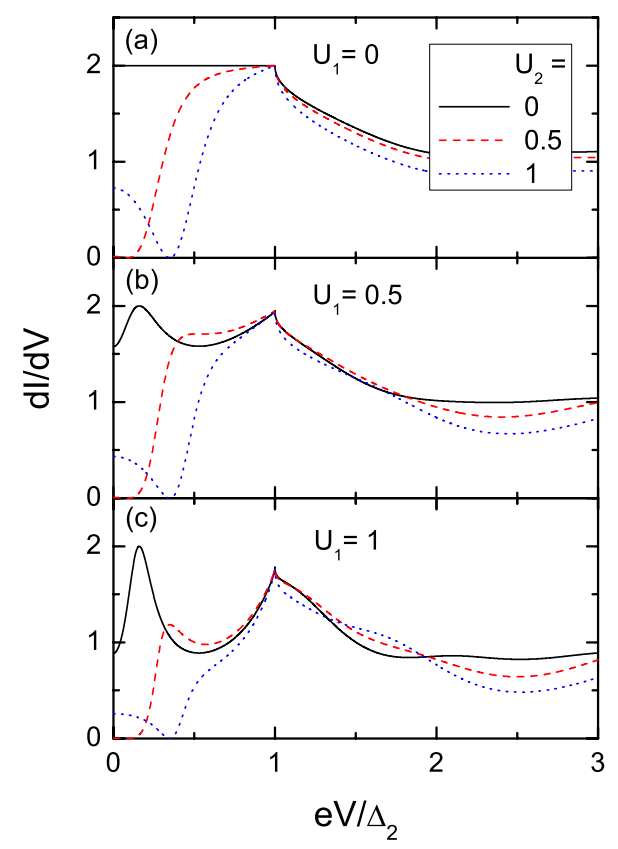

FIG. 4. (Color online) The conductance with various contact barriers. The other parameters are taken as $\Delta_{2}=2 \Delta_{1}=0.001 \mu$ and $d=1.5 \xi_{c 2}$.

from the in-gap bound state exists only for nonzero $U_{1}$ and becomes more pronounced as $U_{1}$ increases. This is because the incoming waves couple to the in-gap bound state only when $U_{1}$ is nonzero.

In the following we shall examine the TRI state in more detail because this is the state likely to be observed in pnictide superconductors. In this case there are two groups of tunneling channels-one with $\theta^{(i)}=0$ and the other with $\theta^{(i)}$ $=\pi$. $S_{1}$ couples strongly to the $\theta^{(i)}=0$ bands (smaller $U_{2}$ ) and has weaker coupling (large $U_{2}$ ) with the $\theta^{(i)}=\pi$ bands. The tunneling conductance is a sum of contributions from all bands. To compute the tunneling spectrum we shall assume that the two groups of bands have the same gap magnitude $\Delta_{2}$ and the $s$-wave superconductor has a weaker gap $\Delta_{1}$ $=0.5 \Delta_{2}$. We shall also assume that $U_{2}\left(\theta^{(i)}=\pi\right)=2 U_{2}\left(\theta^{(i)}=0\right)$. The two bands contribute equally to the conductance in our calculation. As shown in Fig. 5, the conductance peak below $\Delta_{1}$ appears only when $S_{2}$ is a $\pm s$-wave superconductor. The conductance of the $s$-wave superconductor is rather insensitive to $U_{2}$ while the in-gap conductance peak of the $\pm s$-wave superconductor is sensitive to changing $U_{2}$. In particular, smaller $U_{2}$ favors a sharper peak.

Experimentally the tunneling spectrum of a multiband superconductor can be obtained rather straightforwardly from scanning tunnel microscope (STM) experiments. The $s$-wave superconductor can be introduced by coating the STM tip with a thin layer of the $s$-wave superconductor, and our predictions can be tested by performing the experiment with different thicknesses of coated $s$-wave superconductor on

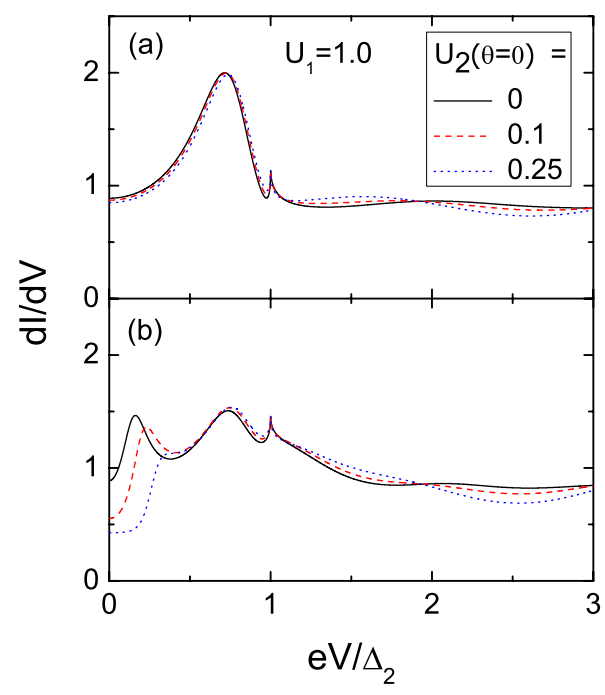

FIG. 5. (Color online) The total conductance when (a) $S_{2}$ is a $s$-wave superconductor and (b) $S_{2}$ is a $\pm s$-wave superconductor. $\Delta_{1}=0.5 \Delta_{2}=0.0005 \mu, U_{1}=\mu$, and $d=1.5 \xi_{c 2}$ in both cases. $U_{2}\left(\theta^{(i)}\right.$ $=\pi)=2 U_{2}\left(\theta^{(i)}=0\right)$ in the case of $\pm s$-wave superconductor.

different surfaces of the targeted superconductor. The tunneling barriers $U_{2}\left(\theta^{(i)}\right)$ can be tuned by varying the distance between the STM tip and the targeted superconductor. The precise values depend also on the tunneling surface orientation.

Nature is much more complicated than what our simple calculation presents which ignores the complicated band structures of the material, ${ }^{15}$ proximity effects, ${ }^{16}$ and scattering between different bands. ${ }^{17}$ The existence of in-gap states in a $S_{1}-N-S_{2}$ junction involving Fe-based superconductors $\left(S_{2}\right)$ is indeed demonstrated in a two-band tight-binding calculation. ${ }^{15}$ We note, however, that the quantitative properties of the tunneling spectrum can be modified by these effects. For example, the gap magnitudes $\Delta_{1}$ and $\Delta_{2}$ close to junction can be modified by proximity effect. ${ }^{16}$ The scattering between different bands in the $\pm s$-wave superconductor due to the junction may also introduce different Andreev bound states for particular orientations of crystal surfaces ${ }^{17}$ which enhances the tunneling conductance. This effect is presumably important when $U_{2}$ is large such that backscattering is strong. Notice however that this is also the regime where the overall tunneling amplitude is suppressed (see Fig. 4). Therefore, the existence of in-gap peaks in the tunneling conductance with sensitive dependence on $d, U_{2}$, but is insensitive to surface orientation provides a strong test to the existence of in-gap states in a $S_{1}-S_{2}$ junction and the gap symmetry of the Fe-based superconductors.

We thank H. H. Wen for interesting discussions during the course of this work. This work was supported by HKUGC through Grant No. CA05/06.Sc04. 
${ }^{1}$ K. Seo, B. A. Bernevig, and J. Hu, Phys. Rev. Lett. 101, 206404 (2008).

${ }^{2}$ I. I. Mazin, D. J. Singh, M. D. Johannes, and M. H. Du, Phys. Rev. Lett. 101, 057003 (2008).

${ }^{3}$ F. Wang, H. Zhai, Y. Ran, A. Vishwanath, and D. Lee, Phys. Rev. Lett. 102, 047005 (2009).

${ }^{4}$ K. Kuroki, S. Onari, R. Arita, H. Usui, Y. Tanaka, H. Kontani, and H. Aoki, Phys. Rev. Lett. 101, 087004 (2008).

${ }^{5}$ C. W. Hicks, T. Lippman, M. Huber, Z. Ren, Z. Zhao, and K. Moler, J. Phys. Soc. Jpn. 78 (1), 013708 (2009).

${ }^{6}$ K. Matano et al., Europhys. Lett. 83, 57001 (2008).

${ }^{7}$ C. Ren, Z. Wang, H. Yang, X. Zhu, L. Fang, G. Mu, L. Shan, and H. Wen, arXiv:0804.1726 (unpublished).

${ }^{8}$ Lin Zhao et al., Chin. Phys. Lett. 25, 4402 (2008).
${ }^{9}$ H. Ding et al., Europhys. Lett. 83, 47001 (2008).

${ }^{10}$ Chia-Ren Hu, Phys. Rev. Lett. 72, 1526 (1994).

${ }^{11}$ Y. Tanaka and S. Kashiwaya, Phys. Rev. Lett. 74, 3451 (1995).

${ }^{12}$ T. K. Ng and N. Nagaosa, arXiv:0809.3343 (unpublished).

${ }^{13}$ P. G. de Gennes, Superconductivity of Metals and Alloys (Benjamin, New York, 1966).

${ }^{14}$ G. E. Blonder, M. Tinkham, and T. M. Klapwijk, Phys. Rev. B 25, 4515 (1982).

${ }^{15}$ See also W.-F. Tsai, D.-X. Yao, A. Bernevig, and J.-P. Hu, arXiv:0812.0661 (unpublished).

${ }^{16}$ A. Brinkman, A. A. Golubov, and M. Yu. Kupriyanov, Phys. Rev. B 69, 214407 (2004).

${ }^{17}$ P. Ghaemi, F. Wang, and A. Vishwanath, Phys. Rev. Lett. 102, 157002 (2009). 